\title{
Le procès qui n'a pas eu lieu
}

Hans Stalder

Correspondance:

Dr Hans Stalder

Professeur honoraire

Chemin Castan 9A

CH-1224 Chêne-Bougeries

johann.stalder@medecine.unige.ch
Le 23 juin 2006, alors chef de service à la Policlinique de médecine et chef au Département de médecine communautaire des Hôpitaux universitaires de Genève (HUG), je suis inculpé par le juge d'instruction pour homicide involontaire avec un de mes collaborateurs. Il m'est reproché $\mathrm{d}^{\prime}$ «avoir omis de donner des directives précises aux médecins [...] quant à la nécessité d'un contrôle du chef de clinique en présence de certaines pathologies»; mon collaborateur, alors médecin consultant à la Policlinique aux urgences, est inculpé pour «avoir, en sa qualité de chef de clinique, laissé (Monsieur R.H.G.) rejoindre son domicile [...].»

Comment sommes-nous arrivés là?

\section{L'histoire du patient}

Le 9 février 2003, donc trois ans et quatre mois avant l'inculpation, M. R.H.G., âgé de 49 ans, se présente aux urgences des HUG. L'infirmière de triage à l'entrée des urgences note comme motif: «Douleur dans la gorge, nez qui coule; a l'impression de ne pas pouvoir bouger ses jambes. Dit avoir fait un faux mouvement avant-hier.» Elle le fait transférer dans le secteur ambulatoire des urgences, dirigé par la Policlinique de médecine.

L'interne note dans son dossier soigneusement rédigé que le patient présente des douleurs lombaires, irradiant dans la jambe droite, survenues à la suite d'un déménagement il y a trois jours, au cours duquel il a porté de lourdes charges. Ces douleurs l'empêchent de marcher plus de dix à quinze mètres sans se reposer une à deux minutes. Il signale en outre souffrir depuis quelque temps de douleurs thoraciques, accentuées à la toux. Il se déclare par ailleurs en bonne santé en dehors d'un problème valvulaire ancien. Il ne fume pas et ne prend pas de médicaments. A l'examen, il présente un souffle systolique; on note des contractures para-vertébrales lombaires importantes. L'examen neurologique des membres inférieurs est normal. La tension artérielle est de 120/60 mm Hg, le pouls à 63/minute. Devant la crainte du patient de présenter un problème cardiaque, l'interne fait un ECG qui dévoile des ondes $\mathrm{T}$ aplaties dans les dérivations inférieures. CPK et troponines sont dans les limites de la norme. Elle conclut à des douleurs thoraciques respiro-dépendantes suite à une toux irritative et une lombo-ischialgie droite. Elle prescrit des anti-inflammatoires et analgésiques ainsi qu'un myorelaxant et laisse partir le patient.

Celui-ci a été vu par ailleurs la veille à un centre médical par une ancienne chef de clinique des HUG qui a posé un diagnostic et lui a remis une prescription similaires.

\section{Procédure judiciaire}

Le 9 septembre 2003, soit 7 mois après la consultation, le juge d'instruction perquisitionne le dossier de R.H.G. auprès du service juridique des HUG. Ni l'interne ni le médecin consultant ne se souviennent du cas. Nous apprenons alors que le patient est décédé trois jours après la consultation, apparemment d'une dissection aortique. C'est la femme du défunt qui se porte plaignante. Une expertise sur les circonstances du décès de R.H.G. est demandée par le juge le 18 novembre 2003 à un professeur cardiologueurgentiste français et au professeur et chef de service de l'Institut universitaire de médecine légale, qui dépend par ailleurs administrativement du Département de médecine communautaire. Le 14 janvier 2004 nous sommes individuellement entendus, ainsi que d'autres personnes impliquées dans ce cas, par les deux experts dans le bureau de mon collaborateur sans que nous ayons accès au rapport anatomo-pathologique. Le 21 juin 2005 et le 13 septembre 2005, soit plus de 16 mois après l'évènement, nous sommes interrogés comme témoins par le juge d'instruction après être libérés des secrets professionnel et de fonction, sans avoir accès ni à l'autopsie ni au rapport des experts. Cet accès nous est accordé seulement après l'inculpation: nous apprenons alors que le patient est décédé d'une rupture d'anévrisme de l'aorte de type A incluant la carotide gauche et les iliaques avec un hémo-péricarde (cause du décès). Il y a en plus une artériosclérose sévère des coronaires. L'expertise conclut à une prise en charge insuffisante. Mon collaborateur porterait une responsabilité directe du fait qu'il n'aurait pas complété l'anamnèse et l'examen clinique et pas demandé des examens complémentaires. A moi, on reproche le manque d'informations aux médecins consultants concernant le niveau de compétence des internes affectés aux urgences et d'instructions claires concer- 
nant leurs responsabilités. L'interne n'est pas accusée de négligence, la bonne tenue du dossier étant mentionnée.

\section{La défense}

Les HUG nous procurent un avocat à chacun. La défense se basera sur le fait de la rareté de l'affection, de la présentation clinique atypique rendant des affections communes beaucoup plus plausibles et l'absence de facteurs de risque. Le reproche fait à mon collaborateur, médecin installé, mais ancien chef de clinique des services de médecine des HUG avec une très grande expérience aux urgences, d'avoir eu besoin d'instructions sur leur fonctionnement et de supervision, semble dérisoire. L'avocat de la compagnie d'assurance de responsabilité des HUG demande par ailleurs deux contre-expertises, une par un professeur de chirurgie cardiaque suisse renommé et l'autre par un expert des urgences. Les deux concluent qu'il n'y a pas eu de négligence.

\section{Une issue surprenante}

L'instruction devant le juge a lieu le 25 septembre 2006 en présence de la plaignante et les avocats. La séance est close après que nous ayons avancé les arguments cités plus haut. Accessoirement, nous relevons à quel point ce cas nous a affectés et qu'avec la meilleure volonté des erreurs ne seront jamais évitées, même dans un centre universitaire.

$\mathrm{Au}$ cours de la séance, l'épouse du défunt nous prend part en éloignant son avocate. En larmes, elle nous fait part de son désarroi, disant qu'elle nous en voulait pas, et nous demande si une issue à l'amiable ne serait pas possible. Nous sommes alors obligés de nous référer à nos avocats respectifs y incluant ceux du service juridique des HUG et de l'assurance. Après cinq mois de discussions et une proposition d'indemnité pour la plaignante, une demande de classement est adressée au procureur général. Celui-ci est accordé le 15 mai 2007, soit 4 ans et 3 mois après la consultation aux urgences.

1 Kohn LT, Corrigan JM, Donaldson MS (eds.). To Err is Human: Building a Safer Health System. Washington, D.C.: National Academic Press; 2000.

2 Hébert PC, Levin AV, Robertson G. Bioethics for clinicians: 23. Disclosure of medical error. CMAJ. 2001; 164:509-13.

3 Genèse 3.8

4 Gallagher $\mathrm{TH}$, Waterman $\mathrm{AD}$, Ebers AG, Fraser VJ, Levinson W. Patients' and physicians' attitudes regarding the disclosure of medical errors. JAMA. 2003;289:1001-7. conséquences sur la santé dans 2,9 à 13,6\% des hospitalisations et le nombre de décès dus à des erreurs de 44000 à 98000 par an aux Etats-Unis. Il est cependant important de souligner que la notion d'erreur est ambiguë: selon le Petit Robert l'«erreur» est définie à la fois comme «acte de l'esprit qui tient pour vrai ce qui est faux et inversement» et comme «chose fausse, erronée, action non prévue par rapport à une norme». La première définition n'implique donc pas nécessairement une faute. Dans les domaines tels que la météorologie ou la médecine les situations sont souvent si complexes et imprévisibles que malgré toutes les précautions une erreur ne peut être évitée. Il peut alors s'agir d'un honorable mistake [2], à distinguer de la vraie faute, sous-entendue dans la deuxième définition où l'erreur serait une déviation de ce qui est généralement acceptable. J'utiliserai la première définition: l'erreur n'implique une faute qu'en cas de déviation de la norme. En médecine, la norme est généralement définie par des guidelines. Etant donné que ceux-ci sont établis sur des bases épidémiologiques, il est évident que les avis sur ce qui est erreur et faute dans un cas particulier peuvent diverger même entre experts; dans des cas extrêmes il incombera à la justice de trancher.

\section{Comment se comporter après une erreur?}

Je ne m'étendrai pas sur la prévention des erreurs car beaucoup d'actions ont été entreprises dans nos institutions (p.ex. les directives sur les incidents et faits graves des HUG). Cependant, il n'existe chez nous guère de directives concernant le comportement après qu'une erreur ait été commise. Le premier réflexe après une erreur humain, car déjà décrit dans la Genèse [3] - est de la cacher. Pour le médecin il est d'autant plus difficile de relever une erreur que le fait de la commettre s'oppose au principe de primum nil nocere. La reconnaissance d'une faute est alors ressentie comme une dévalorisation aussi bien devant les patients que devant ses collègues [4]. Il a cependant été démontré que les patients, contrairement à ce que pensent les médecins, désirent unanimement être informés et ceci pas nécessairement pour des motifs indemnitaires: ils veulent surtout que les mêmes erreurs soient évitées dans le futur pour d'autres personnes; ils désirent une communication honnête même sur les erreurs qui n'ont pas eu de suites néfastes [4]. Il existe aussi des raisons éthiques de communiquer les erreurs [2]: l'omission peut péjorer la relation soignant-soigné, l'action de corriger l'erreur devant être camouflée. Sur le plan légal, le patient a droit à l'information. Si ce droit implique aussi l'information sur des erreurs sans 
5 Fellmann W. Arzt und das Rechtsverhältnis zum Patienten. In: Kuhn MW, Poledna T (Hrsg.). Arztrecht in der Praxis. Zürich: Schulthess; 2007. S. 103-231;134.

6 Kraman SS, Hamm G. Risk management: extreme honesty may be the best policy. Ann Intern Med. 1999;131:970-2.

7 Medical professionalism in the new millennium: a physician charter. Ann Intern Med. 2002; 136:234-6.

8 Lazare A. Apology in medical practice: an emerging clinical skill. JAMA. 2006;296:1401-4.

9 Liebman CB, Hyman CS. A mediation skills model to manage disclosure of errors and adverse events to patients. Health Aff (Millwood). 2004;23:22-32

10 Delbanco T, Bell SK. Guilty, afraid, and alone - struggling with medical error. N Engl J Med. 2007; 357:1682-3.

11 Code de déontologie de la FMH. www.fmh.ch.

12 www.patientensicherheit.ch/ de/publikationen/index.html

13 When things go wrong. Responding to adverse events. A consensus statement of the Harvard hospitals. 2007. www.macoalition.org.

14 Jäger P, Schweiter A. Der Hindsight Bias (Rückschaufehler) - ein grundsätzliches Problem bei der Beurteilung ärztlichen Handeln in Arzthaftpflicht- und Arztstrafprozessen. Schweiz Ärztezeitung. 2005;86(33):1940-3. conséquences reste contesté en Suisse [5]. Souvent, les médecins craignent que l'admission d'une erreur, voire d'une faute pourrait avoir des suites judiciaires [4]. Cette crainte ne semble pas fondée, car les institutions ayant introduit l'obligation de relever toutes les erreurs ont constaté une diminution des plaintes juridiques [6]. Il n'est donc pas étonnant que la charte américaine-européenne du professionnalisme médical publiée en 2002 [7] exige une prompte révélation de toutes les erreurs. Le rôle des institutions ne serait donc pas seulement de s'occuper de la prévention des erreurs, mais aussi de promouvoir la communication aux patients lésés. Le savoir-faire y joue un rôle important [8] et peut être enseigné [9]. Enfin, la prise en charge des soignants qui ont commis une erreur doit être instaurée, car ils se trouvent souvent désemparés, isolés et angoissés et guère préparés d'affronter une procédure juridique [10].

En Suisse nous avons clairement du retard dans ces matières: la FMH ne mentionne pas le comportement en cas d'erreur dans ses directives déontologiques [11] et il n'existe pas de directive nationale concernant les problèmes éthiques des erreurs. Uniquement la Fondation sur la Sécurité des Patients [12] a traduit en allemand les excellentes recommandations des hôpitaux de Harvard aux Etats-Unis (qui peuvent être obtenues gratuitement [13]). Elles n'ont cependant pas été adaptées à notre système de santé, la traduction n'existe pas en français et en surplus est payante ...

\section{L'interaction avec les services juridiques et les autorités judiciaires}

Ces dernières années les services juridiques hospitaliers ont pris une ampleur impressionnante. Sans vouloir mettre en doute leur utilité, notons qu'ils ont tendance à considérer toute plainte immédiatement comme contentieux avant de laisser à un patient mécontent la possibilité de prendre contact avec les soignants impliqués. Dans notre cas, la première rencontre avec l'épouse du défunt n'a eu lieu qu'après l'inculpation, soit plus de trois ans après le décès de son mari. Il nous semble significatif que ce premier contact a été suivi d'un retrait de sa plainte.

Les critiques envers l'autorité judiciaire sont tout aussi sévères. Le dossier aurait pu être saisi plus tôt après le décès suspect: on se serait souvenu du déroulement de la consultation. Si une expertise devient nécessaire, le choix des experts est crucial: un expert doit être impartial et familier avec les circonstances locales. Il doit éviter le hindsight bias (la vue par le «retro-spectroscope») [14]. Dans notre cas, il s'agissait d'un spécialiste cardiologue étranger, guère habitué à un service de premier recours, associé au collaborateur d'un des médecins impliqués, psychiatre de surcroît: il est fort à parier que cette expertise n'aurait pas tenu bonne devant le tribunal! De plus, entendre des personnes d'abord comme témoins pour ensuite les inculper paraît une attitude discutable.

\section{Conclusions}

En conclusion voici quelques constations et propositions:

1. Les erreurs médicales ne pourront jamais être complètement évitées. Ainsi chaque médecin doit être conscient qu'il puisse un jour être confronté à une erreur et à ses conséquences.

2. Un dossier bien fait peut éviter des procédures juridiques.

3. Pour des raisons éthiques chaque erreur devrait être communiquée par les soignants au patient lésé.

4. Le savoir-faire de la communication d'erreurs devrait être enseigné au niveau prégradué par les facultés et au niveau postgradué par les sociétés des spécialités.

5. L'attitude à adopter en cas d'erreur devrait être incluse dans les directives déontologiques de la FMH.

6. Il serait utile de disposer d'une directive nationale établie par l'Académie Suisse des Sciences Médicales (ASSM) concernant les aspects éthiques des erreurs médicales.

7. Les institutions devraient offrir une prise en charge des soignants ayant commis des erreurs.

8. Les services juridiques devraient promouvoir le dialogue entre soignants et personnes lésées avant de s'emparer du dossier et le rendre contentieux. Ce dialogue serait de nature à éviter des procédures judiciaires inutiles, onéreuses et pénibles.

9. Les procédures judiciaires ayant trait aux erreurs médicales devraient être conduites dans les plus brefs délais afin de permettre le rétablissement correct des faits et de ne pas augmenter inutilement l'angoisse et la souffrance des parties impliquées.

10. L'autorité judiciaire devrait choisir soigneusement des experts bien adaptés à l'événement.

Espérons que notre expérience douloureuse et nos commentaires inciteront les institutions hospitalières et judiciaires, ainsi que les facultés de médecine, la FMH et l'ASSM à une réflexion! 